\title{
Spontaneous lateral sphenoid cerebrospinal fluid fistula: MRI diagnosis
}

\author{
Matthew Goodier, MB ChB \\ Darlene Lubbe, \\ Savvas Andronikou, MB BCh, FCRad, FRCR (Lond), PhD \\ Rene Truter, MB ChB, MMed (Radiol) \\ Department of Radiology, University of the Witwatersrand, Johannesburg
}

Corresponding author: M Goodier (goodiermatt@gmail.com)

\begin{abstract}
Spontaneous or primary cerebrospinal fluid (CSF) fistula is a rare cause of CSF rhinorrhoea. Magnetic resonance imaging with highresolution highly $\mathrm{T} 2$-weighted images is valuable in pre-operative localisation and characterisation of the defect, particularly if a transnasal endoscopic approach is planned. This report describes the radiological evaluation and surgical management of a 53-year-old man who presented with a spontaneous lateral sphenoid CSF fistula.
\end{abstract}

\section{Case report}

We present the case of a 53-year-old man who presented with a primary complaint of a lateral tongue tumour. During admission for surgery, a large lesion was noticed on his occiput. An MRI revealed a large occipital meningioma for which he had a subtotal resection. Postoperatively he presented with an unexplained spontaneous CSF leak through the right sphenoid sinus. The patient never had documented raised pressures, and it was felt that the remaining tumour residue could not be responsible for causing raised intracranial pressure.

The MRI performed at the time demonstrated a herniation of meninges through a bony defect in the lateral wall of the right sphenoid sinus and subtle features of encephalomalacia of the adjacent temporal lobe (Figs 1a - c).

An endoscopic transsphenoidal reduction of the meningocoele and obliteration of the right sphenoid sinus was performed.
At surgery, a $1 \mathrm{x} 1 \mathrm{~cm}$ dehiscent area in the right sphenoid sinus was present. There was some mucosal thickening with visible dura which was cauterised to identify the actual leak. The sinus was cleared of all mucosa and obliterated with fat. Six weeks post-operatively, the patient was well, with no further CSF leak.

\section{Discussion}

The presence of CSF rhinorrhoea indicates the existence of an abnormal communication between the intracranial CSF spaces and the nasal cavity (or via the eustachian tube from a temporal bone fracture and CSF-leak). Post-traumatic CSF rhinorrhoea as a complication of a base of skull fracture is the most common cause. Non-traumatic causes of CSF rhinorrhoea make up only $3-4 \%$ of cases $^{1}$ and comprise several causes such as skull base tumours, destructive granulomatous processes such as Wegener's granulomatosis, and complicated sinonasal infections. ${ }^{1}$

Spontaneous, or primary, CSF fistula is a separate entity with no underlying cause of the CSF leak. Spontaneous leaks are more common in obese middle-aged women, and there is commonly a co-existing encephalocele of variable size. ${ }^{2}$ The pathogenesis of this condition is thought to be due to a combination of embryological and acquired factors. The medial aspect of the temporal bone may fail to develop, resulting in a persistent lateral craniopharyngeal canal (also known as the Sternberg canal) between the middle cranial fossa and the pneumatised inferolateral recess of the sphenoid sinus. ${ }^{3}$ Idiopathic intracranial hypertension (pseudotumour cerebri) and empty sella syndrome are also known to occur in these patients, ${ }^{2}$ suggesting that

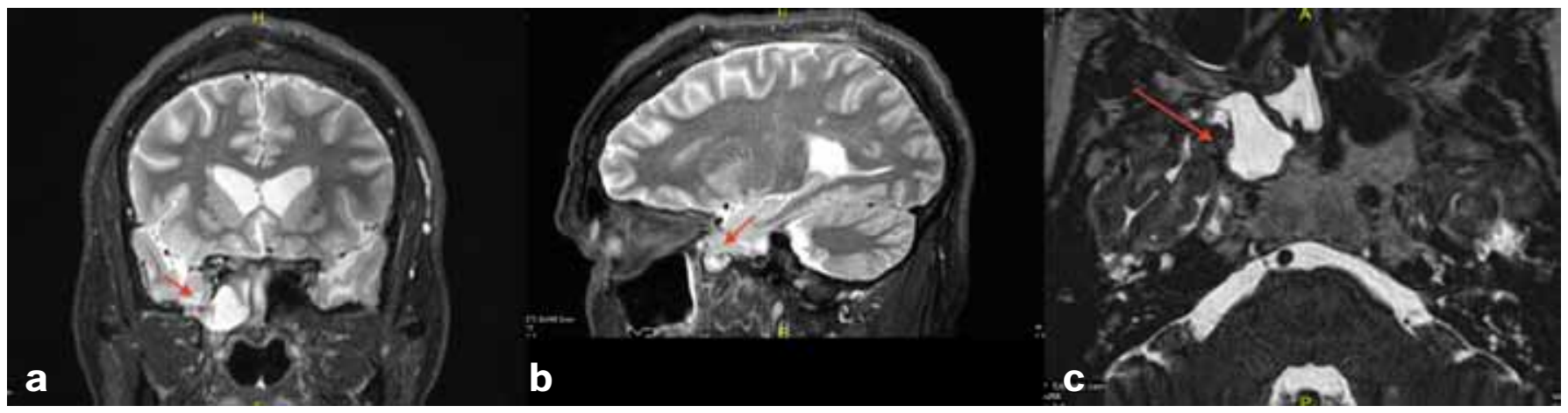

Figs $1 a-1 c$. MRI scans of the brain and sphenoid sinus in a patient with lateral sphenoidal CSF fistula. (1a) Coronal (1b)sagittal and (1c) high-resolution axial T2-weighted images demonstrate a herniation of CSF-filled meninges from the right middle temporal fossa into the right sphenoid sinus through a bony defect (arrow). There is subtle encephalomalacia of the right temporal pole. 
chronically increased intracranial pressure with localised thinning of the bone may also play a role in the pathogenesis of this condition. ${ }^{4}$

Spontaneous CSF fistulae are most common in the anterior cranial fossa, at the ethmoid roof and cribriform plate. Less common sites include the sphenoid sinus, around the sella or at the inferolateral or pterygoid recesses. $^{2}$

The goals of imaging in CSF fistulae are to confirm the diagnosis, evaluate any underlying cause, localise the defect site and exclude an associated meningoencephalocoele at the defect. ${ }^{2}$ Successful treatment depends on the accurate pre-operative localisation of the site of the fistula. This is especially true as most defects are now repaired by means of endoscopic surgery. An open approach via a craniotomy may be required in large defects or in well pneumatised sphenoid sinuses where the defect is located in the lateral recess of the sphenoid sinus. Pure obliteration of the sphenoid sinus would rarely be successful in large defects, and a vascularised flap is often needed in these instances. The mucoperichondrial septal flap, pedicled on the posterior septal artery, is a useful flap when endoscopic repair is considered.

High-resolution CT scanning alone has a low sensitivity for the detection of the osseous-dural defect. Accuracy may be increased by introducing low-oslomar contrast materal into the subarachnoid space (CT cisternography), which improves the sensitivity for active leaks to $80-85 \% .^{3}$ Inactive or intermittent fistulae may not be demonstrated, however. CT is best for bony anatomical detail and is useful in preoperative planning.

Magnetic resonance imaging (MRI) provides complementary information and appears to have a higher sensitivity than CT scanning, with accuracy rates for detection of the site of even inactive fistulae as high as $100 \%{ }^{5}$ High-resolution highly T2-weighted images such as are obtained by the constructive interference in the steady state (CISS) sequence are the most useful. MRI is also able to depict the contents of any associated encephalocoele. Subtle encephalomalacia as seen in our patient is often present at the site of the leak. ${ }^{2}$ MRI was sufficient to allow accurate pre-operative assessment in our patient.

Surgery for spontaneous CSF leaks has traditionally been performed transcranially. Success rates of up to $80 \%{ }^{2}$ have been reported; however, there is additional morbidity related to the craniotomy as well as the risk of anosmia owing to damage to the olfactory tracts.

Transnasal endoscopic surgical techniques are now increasingly performed, especially for small defects. ${ }^{6}$ In experienced hands, the reported success rates approach $90 \%$, which is superior to open repair. ${ }^{2}$ Some cases, however, are not amenable to endoscopic techniques; these include high-pressure leaks, patients with multiple defects, large defects or very well pneumatised sphenoid sinuses with lateral recesses. ${ }^{2}$

\section{Conclusion}

Spontaneous or primary CSF fistula is a rare cause of CSF rhinorrhoea. Various imaging modalities including CT, CT cisternography, MRI and radio-isotope scanning may be used for preoperative evaluation. MRI with high-resolution and highly T2-weighted images are most valuable in preoperative localisation and characterisation of the defect, especially if a transnasal endoscopic approach is planned.

1. Pandey AK. Case report: Anteromedial temporosphenoidal encephalocele with a clinically silent lateral bony defect in the greater wing of the sphenoid. Indian J Radiol Imaging 2009;19:311-313.

2. Lloyd KM, DelGaudio JM, Hudgins PA. Imaging of skull base cerebrospinal fluid leaks in adults. Radiology 2008;248:725-736.

3. Wind JJ, Caputy AJ, Roberti F. Spontaneous encephaloceles of the temporal lobe. Neurosurg Focus 2008;25:1-6.

4. Schuknecht B, Simmen D, Briner HR, Holzmann D. Nontraumatic skull base defects with spontaneous CSF rhinorrhea and arachnoid herniation: imaging findings and correlation with endoscopic sinus surgery in 27 patients. Am J Neuroradiol 2008;29:542-549.

5. Johnson DB, Brennan P, Toland J, O'Dwyer AJ. Magnetic resonance imaging in the evaluation of cerebrospinal fluid fistulae. Clin Radiol 1996;51:837-841.

6. Arai A, Mizukawa K, Nishihara M, Fujita A, Hosoda K, Kohmura E. Spontaneous cerebrospinal fluid rhinorrhea associated with a far lateral temporal encephalocele - case report. Neurol Med Chir (Tokyo) 2010;50:243-245. 